LPT-ORSAY-08-26

October 27, 2018

\title{
The constrained next-to-minimal supersymmetric standard model
}

\author{
A. Djouadi ${ }^{1,2,3}$, U. Ellwanger $^{1}$ and A.M. Teixeira ${ }^{1}$ \\ ${ }^{1}$ Laboratoire de Physique Théorique, CNRS and Université Paris-Sud, \\ F-91405 Orsay Cedex, France. \\ ${ }^{2}$ School of Physics and Astronomy, Southampton University, Highfield, SO17 1BJ, UK. \\ ${ }^{3}$ Physikalisches Institute, University of Bonn, Nussallee 12, 53115 Bonn, Germany.
}

\begin{abstract}
We consider the fully constrained version of the next-to-minimal supersymmetric extension of the standard model (cNMSSM) in which a singlet Higgs superfield is added to the two doublets that are present in the minimal extension (MSSM). Assuming universal boundary conditions at a high scale for the soft supersymmetrybreaking gaugino, sfermion and Higgs mass parameters as well as for the trilinear interactions, we find that the model is more constrained than the celebrated minimal supergravity model. The phenomenologically viable region in the parameter space of the cNMSSM corresponds to a small value for the universal scalar mass $m_{0}$ : in this case, one single input parameter is sufficient to describe the phenomenology of the model once the available constraints from collider data and cosmology are imposed. We present the particle spectrum of this very predictive model and discuss how it can be distinguished from the MSSM.
\end{abstract}


The next-to-minimal supersymmetric standard model (NMSSM) [1-3], in which the spectrum of the minimal extension (MSSM) is extended by one singlet superfield, was among the first supersymmetric (SUSY) models based on supergravity-induced SUSYbreaking terms. It has gained a renewed interest in the last decade, since it solves in a natural and elegant way the so-called $\mu$ problem [4] of the MSSM; in the NMSSM this parameter is linked to the vacuum expectation value (vev) of the singlet Higgs field, generating a value close to the SUSY-breaking scale. Furthermore, it leads to an interesting phenomenology as the MSSM spectrum is extended to include an additional CP-even and CP-odd Higgs state as well as a fifth neutralino, the singlino.

In contrast to the non- or partially constrained versions of the NMSSM that have been intensively studied in the recent years [5], and which involve many free parameters, the constrained model (cNMSSM) has soft SUSY-breaking parameters that are universal at a high scale. This is motivated by schemes for SUSY-breaking that are mediated by (flavor blind) gravitational interactions, and leads to a more predictive model as the number of unknown parameters is reduced to a handful.

In the constrained MSSM or minimal supergravity (mSUGRA) scenario [6], the universality of the soft parameters at the grand unification scale $M_{\mathrm{GUT}}$ leads to only four continuous parameters: the common gaugino $M_{1 / 2}$ and scalar $m_{0}$ masses, the trilinear coupling $A_{0}$, and the ratio of vevs of the two Higgs fields $\tan \beta$. (The conditions for a correct electroweak symmetry breaking allow to trade the two basic Lagrangian parameters $\mu^{2}$ and its corresponding soft breaking term $B \mu$ by the $Z$ boson mass $M_{Z}$ and $\tan \beta$, leaving the sign of $\mu$ undetermined.) The numerous soft parameters at low energies are then obtained through renormalization group (RG) evolution. The cNMSSM with universal soft terms at the GUT scale has the same number of unknown parameters: instead of the cMSSM $\mu$ and $B$ parameters, one has the trilinear couplings $\lambda$ and $\kappa$ in the Higgs sector.

General features of the cNMSSM parameter space as well as its phenomenology have been discussed in Refs. [2,3]. These studies already revealed that the allowed range for the parameters $M_{1 / 2}, m_{0}$ and $A_{0}$ is different from that of the cMSSM. Indeed while small values for $m_{0}$ are disfavored in the cMSSM as they lead to charged sleptons that are lighter than the neutralino $\chi_{1}^{0}$, which is the preferred lightest SUSY particle (LSP), small $m_{0}$ is needed in the cNMSSM to generate a non-vanishing vev of the singlet Higgs field [2]; the slepton LSP problem can be evaded owing to the presence of the additional singlino-like neutralino which, in large regions of the cNMSSM parameter space, is the true LSP. Note that vanishing values for $m_{0}$ are naturally obtained in supergravity models with Kähler potentials of the no-scale type [7]; however, the additional no-scale prediction $A_{0}=0$ is difficult to realize in the cNMSSM.

Since the early studies, bounds on the Higgs and SUSY particle spectrum from highenergy collider data and low-energy measurements have become more severe, while important inputs such as the top quark mass are more accurately measured [8]. In addition, 
tools for a more precise determination of the mass spectrum and couplings [9], and the cosmological dark matter relic density [10] have become available. In this letter, we reinvestigate the parameter space of the cNMSSM in the light of these recent constraints, using the updated tools.

We find a viable region of the cNMSSM parameter space very close to the no-scale situation: vanishing or very small $m_{0}$ and $\left|A_{0}\right|$ well below $M_{1 / 2}$. In fact, in order to obtain the correct cosmological relic density for the LSP, which turns out to be a singlinolike neutralino [11], $A_{0}$ is essentially fixed in terms of $M_{1 / 2} ; \tan \beta$ is determined by the scalar mass universality (including the additional singlet), while LEP constraints on the Higgs sector imply $\lambda \lesssim 10^{-2}$ and this parameter has practically no effect on the remaining particle spectrum.

Thus, remarkably, only one single parameter is sufficient to describe the phenomenological features of the cNMSSM. This makes the model much more predictive than the celebrated mSUGRA model. In addition, as will be shown, the phenomenology differs considerably in the two scenarios.

In this analysis, we consider the NMSSM with a scale invariant superpotential given by

$$
\mathcal{W}=\lambda S H_{u} H_{d}+\frac{\kappa}{3} S^{3}+\ldots
$$

where the two terms shown substitute the $\mu H_{u} H_{d}$ term in the MSSM superpotential and we have omitted the usual generalization of the Yukawa interactions. The soft SUSYbreaking terms consist of mass parameters for the gauginos $M_{1,2,3}$, sfermions $m_{\tilde{F}_{L, R}}$ and Higgs fields $m_{H_{u, d}}$ and trilinear interactions $A_{f}$ as in the MSSM, supplemented by an additional scalar mass $m_{S}$ and two trilinear couplings $A_{\kappa}$ and $A_{\lambda}$ for the singlet field. Once the unification of these soft SUSY-breaking masses and trilinear couplings at the scale $M_{\mathrm{GUT}}$ is imposed, the Lagrangian of the cNMSSM depends on the five parameters, $M_{1 / 2}, m_{0}, A_{0}, \lambda$ and $\kappa$. The correct value for $M_{Z}$ reduces the dimension of the parameter space from five to four; e.g. $\kappa$ can be determined in terms of the other parameters. Hence, the number of continuous free parameters in the cNMSSM is the same as in the cMSSM.

For practical purposes it is convenient to adopt the following procedure: In addition to $M_{Z}$, one allows for the five cNMSSM input parameters

$$
M_{1 / 2}, m_{0}, A_{0}, \lambda \text { and } \tan \beta \text {. }
$$

The parameters $\kappa$ and the soft singlet mass squared $m_{S}^{2}$ are both determined at the scale $M_{\text {SUSY }}$ through the minimization equations of the scalar potential. (The vev $\langle S\rangle$ or $\mu_{\text {eff }} \equiv$ $\lambda\langle S\rangle$ is also fixed through the third independent minimization equation, which leaves the sign of $\mu_{\text {eff }}$ undetermined.) This is the procedure employed by the routine NMSPEC within NMSSMTools [9], which calculates the spectra of the Higgs and SUSY particles in the NMSSM in terms of the soft SUSY-breaking terms at $M_{\mathrm{GUT}}$ (except for the parameter $\left.m_{S}^{2}\right), \tan \beta$ at the weak scale and $\lambda$ at the SUSY-breaking scale $M_{\mathrm{SUSY}}$. 
Clearly, the soft singlet mass squared $m_{S}^{2}$ at $M_{\mathrm{GUT}}$ will in general not coincide with $m_{0}^{2}$. However, one can confine oneself to regions in parameter space where the difference between $m_{S}^{2}$ and $m_{0}^{2}$ is negligibly small. This condition leaves us with an effective fourdimensional parameter space, consistent with the considerations above.

Let us now explore the cNMSSM parameter space defined by arbitrary values of $M_{1 / 2}$, $A_{0}$ and $\lambda$, assuming $\mu_{\text {eff }}$ positive and, for the time being, restricting ourselves to $m_{0}=0$. For each choice of $M_{1 / 2}, A_{0}$ and $\lambda, \tan \beta$ is determined by the requirement that $m_{S}^{2}$ at $M_{\mathrm{GUT}}$ should be close to $m_{0}^{2}=0$. In practice, we impose $m_{S}^{2}\left(M_{\mathrm{GUT}}\right)<(5 \mathrm{GeV})^{2}$, which typically requires to tune the fourth decimal of $\tan \beta$ (this should not be interpreted as a finetuning, since $m_{S}^{2}$ should be considered as an input parameter, whereas $\tan \beta$ is determined by the minimization of the effective potential). For the most relevant SM parameters we chose $\alpha_{s}\left(M_{Z}\right)=0.1172, m_{b}\left(m_{b}\right)^{\overline{\mathrm{MS}}}=4.214 \mathrm{GeV}$ and $m_{\mathrm{top}}^{\text {pole }}=171.4 \mathrm{GeV}[8]$.

For this set of parameters, we select the cNMSSM space which survives once one imposes theoretical requirements such as correct electroweak symmetry breaking, perturbative couplings at the high scale, the absence of tachyonic masses, a neutralino LSP, etc.., and phenomenological constraints such as the LEP bounds on Higgs masses and couplings, collider bounds on the SUSY particle masses [8], experimental data from $B$ meson physics [12] and from the anomalous magnetic moment of the muon [13], and a relic density compatible with cosmological data [14]. For $\lambda \lesssim 10^{-2}$, as it turns out to be the case (see below), the phenomenologically allowed region is nearly independent of the input $\lambda$.

Leaving aside, for the time being, the WMAP constraints on the LSP relic density $\Omega h^{2}$, but including all the other constraints above, the phenomenologically allowed region in the $\left[M_{1 / 2}, A_{0}\right]$ plane is shown in Fig. 1.

This allowed region is bounded from below, i.e. for large absolute values of $\left|A_{0}\right|$, by the absence of a charged (generally tau slepton) LSP as in the cMSSM for $m_{0}=0$; inside the allowed region, the LSP is a singlino-like state. The upper bound at $A_{0} \sim 0$ follows from the positivity of the mass squared of the singlet-like CP-odd Higgs boson, which is given to a good approximation by $-3 \kappa A_{\kappa}\langle S\rangle, \kappa\langle S\rangle$ being positive, and $A_{\kappa} \sim A_{0}$. To the left, i.e. towards smaller values of $M_{1 / 2}$, the allowed region is bounded simultaneously by the condition that the lightest tau slepton mass must be above $\sim 100 \mathrm{GeV}$ from its non-observation at LEP, and the mass of the lightest SM-like CP-even Higgs boson above $\sim 114 \mathrm{GeV}$. To the right, i.e. towards larger values of $M_{1 / 2}$ for which SUSY particles are too heavy to be produced at the LHC, no "physical" constraint appears. We believe, however, that this region requires more and more hidden finetuning 1 . Inside this allowed region, $\tan \beta$ is fixed by the requirement $m_{S}^{2}\left(M_{\mathrm{GUT}}\right) \sim 0$, and turns out to be quite large (see Ref. [15] for earlier work on the cNMSSM at large $\tan \beta$ ). In Fig. 1, we have indicated lines corresponding to constant $\tan \beta=25,30,33$ and 35 .

\footnotetext{
${ }^{1}$ The actual finetuning is hidden by our procedure which determines $\kappa$ in terms of other parameters such as $M_{Z}$; this is similar to the determination of $B$ in the cMSSM.
} 


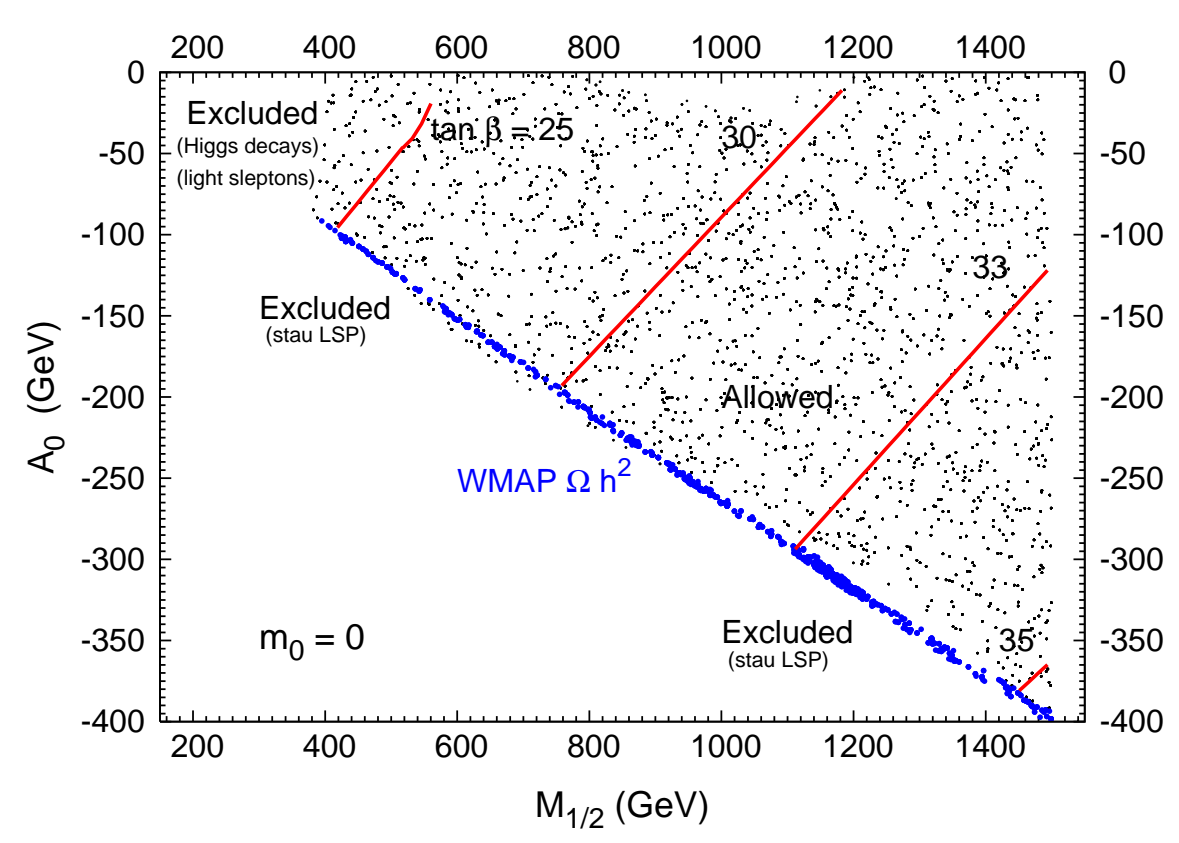

Figure 1: The viable cNMSSM region in the $\left[M_{1 / 2}, A_{0}\right]$ plane for $m_{0} \sim 0$ and $\lambda=2 \times 10^{-3}$, once theoretical, collider and cosmological constraints have been imposed.

As a next step, we require that the WMAP constraint on the relic density of the $\chi_{1}^{0}$ dark matter (DM) candidate, calculated using the program MicrOMEGAS [10], is satisfied. Given the actual small error bars, $0.094 \lesssim \Omega_{\chi_{1}^{0}} h^{2} \lesssim 0.136$ at the $2 \sigma$ level [14], this constraint (if satisfied at all) reduces the parameter space of any model to a lower dimensional hypersurface. Within the allowed region in Fig. 1, the correct relic density for $\chi_{1}^{0}$ is obtained along the line close to the lower boundary (DM line), where the mass of the singlino-like LSP is close to the mass of the next-to-LSP which is the lightest tau slepton $\tilde{\tau}_{1}, M_{\tilde{\tau}_{1}}-M_{\chi_{1}^{0}} \sim 3$ to $5 \mathrm{GeV}$. Near the upper boundary, the mass of the singlino-like LSP becomes very small, implying a far too large relic density. For $\lambda \ll 1$ (see below), the singlino-like $\chi_{1}^{0}$ LSP has very small couplings to SM particles and, thus, the main process which generates the correct relic density is the assisted annihilation or "co-annihilation" of tau sleptons, $\tilde{\tau}_{1} \tilde{\tau}_{1} \rightarrow$ SM particles, which does not depend on these coupling 2 .

As mentioned previously, we have checked that constraints from $B$-physics [12], such as the branching ratio for the radiative decays $b \rightarrow s \gamma$, are satisfied. Moreover, we find that the supersymmetric contribution to the anomalous magnetic moment of the muon, $\delta a_{\mu}=(g-2)_{\mu}$, accounts for the $\sim 3 \sigma$ deviation from the SM expectation [13]: along the DM line, $\delta a_{\mu}$ decreases from $\sim 4.2 \times 10^{-9}$ for $M_{1 / 2} \sim 400 \mathrm{GeV}$ to $\sim 0.2 \times 10^{-9}$ for $M_{1 / 2} \sim 1.5 \mathrm{TeV}$. In view of a desired value $\delta a_{\mu} \sim(2.7 \pm 2) \times 10^{-9}$, the region $M_{1 / 2} \lesssim 1 \mathrm{TeV}$ is thus preferred by this observable.

\footnotetext{
${ }^{2}$ However, $\lambda$ values larger than $\sim 10^{-5}$, as used here, are needed such that the hypothesis of thermal equilibrium between the LSP and the next-to-LSP near the relevant temperature can be considered satisfactory.
} 
At this stage, one can conclude that for small $m_{0}$ and $\lambda$, only one single parameter is needed to describe the phenomenology of the cNMSSM, once collider and cosmological constraints are imposed. Along the DM line in Fig. 1, once a value for $M_{1 / 2}$ is chosen, both the universal trilinear coupling $A_{0}$ and $\tan \beta$ are uniquely fixed. Before investigating the impact of other values for the parameters $m_{0}$ and $\lambda$, let us first discuss the Higgs and sparticle spectrum as a function of $M_{1 / 2}$ in the phenomenologically allowed region. The Higgs, neutralino and stau masses are shown in Fig. 2 where we also indicate the corresponding values of $A_{0}$.

The essential features of the Higgs spectrum are as follows. For $M_{1 / 2} \lesssim 660 \mathrm{GeV}$, the lightest CP-even Higgs boson has a dominant singlet component, hence a very small coupling to the $Z$ boson, which allows it to escape LEP constraints. The next-to-lightest CP-even scalar is SM-like, with a mass slightly above $114 \mathrm{GeV}$. The lightest CP-odd scalar is again singlet-like, with a mass above $\sim 120 \mathrm{GeV}$. The heaviest CP-even and CP-odd scalars are practically degenerate with the charged Higgs boson, with masses above $\sim 520$ GeV. For $M_{1 / 2} \gtrsim 660 \mathrm{GeV}$, the lightest CP-even scalar is SM-like with a mass increasing slightly with $M_{1 / 2}$ up to $\sim 120 \mathrm{GeV}$, while the next-to-lightest one is now singlet-like.

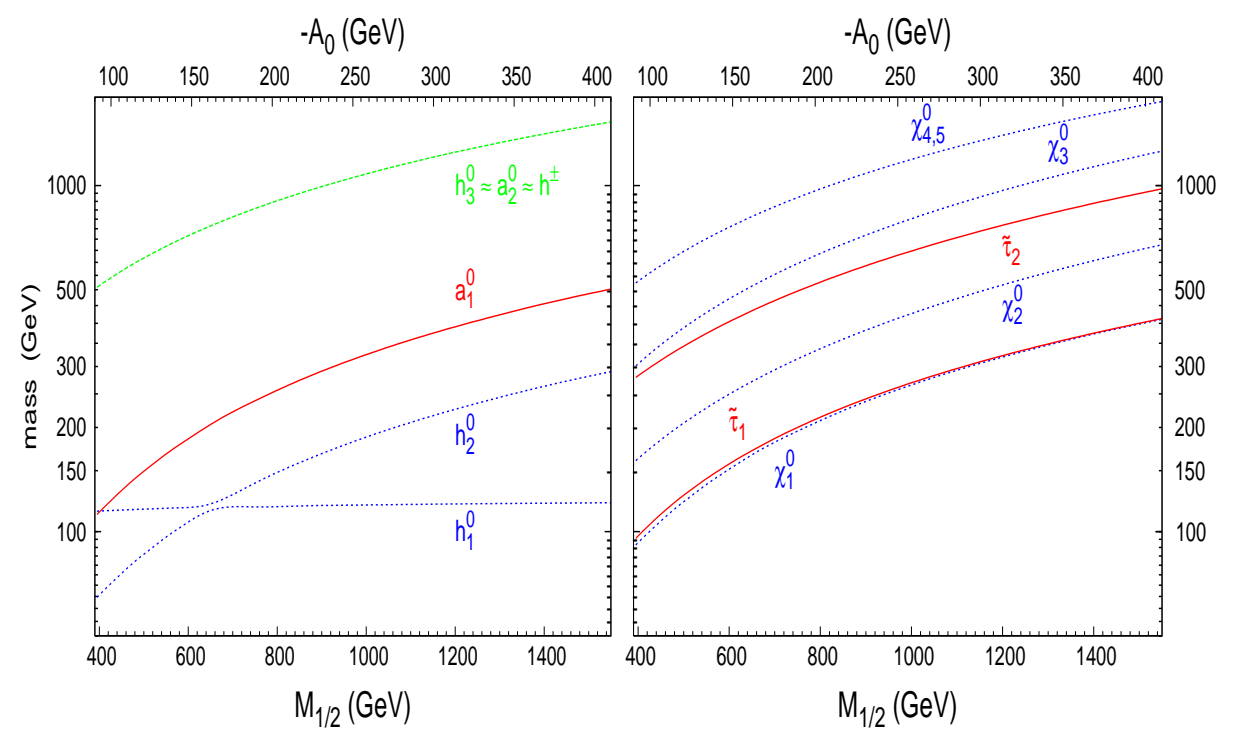

Figure 2: The Higgs (left) and neutralino plus stau (right) mass spectra in GeV as a function of $M_{1 / 2}$ along the DM line; the values of $A_{0}$ are indicated in the upper axis.

The right-hand side of Fig. 2 displays the neutralino and stau spectrum, the lighter stau $\widetilde{\tau}_{1}$ being the next-to-LSP with a mass $\sim 3$ to $5 \mathrm{GeV}$ above the one of the $\chi_{1}^{0}$ singlinolike LSP. $\chi_{2}^{0}$ and $\chi_{3}^{0}$ are, respectively, bino and wino-like while the nearly degenerate $\chi_{4,5}^{0}$ states are Higgsino-like. The charginos $\chi_{1}^{ \pm}$and $\chi_{2}^{ \pm}$are nearly degenerate in mass with, respectively, $\chi_{3}^{0}$ and $\chi_{4,5}^{0}$ (notice that the mass of the latter is $\sim \mu_{\text {eff }}$ ). The remaining sparticle spectrum is very "cMSSM"-like and can be obtained by running the program NMSPEC [9] with input parameters as in Fig. 3 below (and $m_{0} \approx 0$ ) and also by using any cMSSM-based code, since the singlet sector practically decouples from the SUSY 
spectrum. One approximately obtains $m_{\tilde{g}} \approx m_{\tilde{q}} \approx 2 M_{1 / 2}$ for the gluino and (first/second generation) squark masses.

Let us now discuss the impact of other values of the parameters $m_{0}$ and $\lambda$. As already stated above, the Higgs and sparticle spectra change very little with $\lambda$ provided that $\lambda$ remains small enough. Upper bounds on $\lambda$ result from LEP constraints on Higgs scalars with masses below the SM limit of $\sim 114 \mathrm{GeV}$. For $M_{1 / 2} \lesssim 660 \mathrm{GeV}$, increasing $\lambda$ increases the mixing of the singlet-like CP-even scalar with doublet-like CP-even scalars and hence its couplings to the $Z$ boson, which must not be too large. For $M_{1 / 2} \gtrsim 660 \mathrm{GeV}$, a stronger mixing among the CP-even scalars can lower the mass of the lighter Higgs boson which is now SM-like, until it violates the LEP bound.



Figure 3: $\tan \beta$ and the upper bound on $\lambda$ as a function of $M_{1 / 2}$ along the DM line.

Fig. 3 shows the corresponding upper limits on the parameter $\lambda$, which are particularly strong in the "crossover"-region near $M_{1 / 2} \sim 660 \mathrm{GeV}$, where relatively small values of $\lambda$ can generate a large mixing angle; in all cases, one has $\lambda \lesssim 0.02$. For completeness, we also show the values of $\tan \beta$ along the DM line.

We have also investigated the cNMSSM parameter space for non-zero $m_{0}$ and found regions which comply with all phenomenological constraints, including the DM relic density. However, in order to generate a non-vanishing vev of the singlet, the inequality $m_{0} \lesssim \frac{1}{3}\left|A_{0}\right|$ has to hold [2] and, thus, solutions only exist for small $m_{0}$. Even for $M_{1 / 2} \approx 1.5 \mathrm{TeV}$, i.e. for squark and gluino with masses of order $3 \mathrm{TeV}$ and already beyond the LHC reach, only values for $m_{0}$ below $\approx 140 \mathrm{GeV}$ are allowed. Smaller $M_{1 / 2}$ corresponds to smaller $A_{0}$ and, for instance, only $m_{0} \lesssim 20 \mathrm{GeV}$ is viable for $M_{1 / 2} \approx 400 \mathrm{GeV}$. Since $m_{0} \ll M_{1 / 2}$ in all cases, we expect the phenomenology of the model to be similar to the one discussed here. More details will be given in a forthcoming publication [18]. 
Finally, let us note that we have also verified possible instabilities of the potential along charge and colour breaking directions in field space, which could be dangerous for $m_{0} \approx 0[2,16]$. Using analytic approximations to the potential along such dangerous directions, the inequality $\left(A_{0}-\frac{1}{2} M_{1 / 2}\right)^{2} \lesssim 9 m_{0}^{2}+\frac{8}{3} M_{1 / 2}^{2}$ was derived in Ref. [2], and is satisfied within the allowed region. Concerning directions in field space in which the potential is unbounded from below, it was shown in Ref. [17] that even if they exist, the decay rate of the standard vacuum is usually much larger than the age of the Universe.

In conclusion, we have shown that the NMSSM with universal boundary conditions at the GUT scale is a very constrained scenario. For small values of the universal scalar mass $m_{0}$, which are theoretically interesting and excluded in the cMSSM, all present collider constraints on sparticle and Higgs masses are satisfied. Moreover, the requirement of a correct relic density for the dark matter candidate further constrains the parameter space to a one-dimensional $\left[M_{1 / 2}, A_{0}\right]$ line. Thus, only one single parameter, which can be taken as $M_{1 / 2}$, is required to describe the salient features of the model.

This model leads to an interesting phenomenology. For large $M_{1 / 2}$, the lightest CP-even Higgs boson is SM-like with a mass smaller than $\sim 120 \mathrm{GeV}$. For small $M_{1 / 2}$, the lightest CP-even Higgs boson is a possibly very light singlet-like state which will, however, be very difficult to detect given the small value of $\lambda$. In the SUSY sector, the singlino-like LSP will considerably modify the decay chains of sparticles [19]: one expects that all sparticles decay via the lightest tau slepton which then decays into the singlino-like LSP, leading to missing energy. For very small $\lambda$, the lifetime of the tau slepton can become so large that its track can be visible [19]. In any case sparticle decays will differ in a spectacular way from the ones expected within MSSM-typical scenarios, which will allow to test the present scenario hopefully in the near future at the LHC.

Acknowledgments: AD is grateful to the Leverhulme Trust (UK) and to the Alexander von-Humboldt Foundation (Germany). We acknowledge support from the French ANR project PHYS@COL\&COS and discussions with S.F. King and S. Moretti.

\section{References}

[1] H.P. Nilles, M. Srednicki and D. Wyler, Phys. Lett. B 120 (1983) 346; J.M. Frere, D.R. Jones and S. Raby, Nucl. Phys. B 222 (1983) 11; J. Ellis, J. Gunion, H. Haber, L. Roszkowski and F. Zwirner, Phys. Rev. D 39 (1989) 844; M. Drees, Int. J. Mod. Phys. A 4 (1989) 3635.

[2] U. Ellwanger, M. Rausch de Traubenberg and C.A. Savoy, Phys. Lett. B 315 (1993) 331; Z. Phys. C 67 (1995) 665 and Nucl. Phys. B 492 (1997) 307.

[3] T. Elliott, S.F. King and P. White, Phys. Lett. B 351 (1995) 213; S.F. King and P. White, Phys. Rev. D 52 (1995) 4183. 
[4] J.E. Kim and H.P. Nilles, Phys. Lett. B 138 (1984) 150.

[5] For a recent discussion and more references, see A. Djouadi et al., arXiv:0801.4321 [hep-ph].

[6] A.H. Chamseddine, R. Arnowitt and P. Nath, Phys. Rev. Lett. 49 (1982) 970; R. Barbieri, S. Ferrara and C. Savoy, Phys. Lett. B 119 (1982) 343; L. Hall, J. Lykken and S. Weinberg, Phys. Rev. D 27 (1983) 2359.

[7] A. B. Lahanas and D. V. Nanopoulos, Phys. Rept. 145, 1 (1987); N. Dragon, U. Ellwanger and M. G. Schmidt, Prog. Part. Nucl. Phys. 18 (1987) 1.

[8] Particle Data Group [W.-M. Yao et al.], J. Phys. G 33 (2006) 1.

[9] U. Ellwanger, J. F. Gunion and C. Hugonie, JHEP 0502 (2005) 066; U. Ellwanger and C. Hugonie, Comput. Phys. Commun. 175 (2006) 290 and Comput. Phys. Commun. 177 (2007) 399; (see also the web site http://www.th.u-psud.fr/NMHDECAY/nmssmtools.html).

[10] G. Belanger, F. Boudjema, C. Hugonie, A. Pukhov and A. Semenov, JCAP 0509 (2005) 001; G. Belanger, F. Boudjema, A. Pukhov and A. Semenov, Comput. Phys. Commun. 174 (2006) 577.

[11] C. Hugonie, G. Belanger and A. Pukhov, JCAP 0711 (2007) 009.

[12] G. Hiller, Phys. Rev. D 70 (2004) 034018; F. Domingo and U. Ellwanger, JHEP 0712 (2007) 090.

[13] G. Bennett et al., Phys. Rev. D 73 (2006) 072003; for a recent review see Z. Zhang, arXiv:0801.4905 [hep-ph].

[14] D. N. Spergel et al. [WMAP Collaboration], Astrophys. J. Suppl. 170 (2007) 377.

[15] B. Ananthanarayan and P.N. Pandita, Phys. Lett. B 353 (1995) 70; Phys. Lett. B 371 (1996) 245; Int. J. Mod. Phys. A 12 (1997) 2321.

[16] U. Ellwanger and C. Hugonie, Phys. Lett. B 457 (1999) 299.

[17] S. A. Abel and C. A. Savoy, Nucl. Phys. B 532 (1998) 3.

[18] A. Djouadi, U. Ellwanger and A.M. Teixeira, in progress.

[19] F. Franke and H. Fraas, Z. Phys. C 72 (1996) 309; U. Ellwanger and C. Hugonie, Eur. Phys. J. C 5 (1998) 723 and Eur. Phys. J. C 13 (2000) 681; V. Barger, P. Langacker and G. Shaughnessy, Phys. Lett. B 644 (2007) 361 and Phys. Rev. D 75 (2007) 055013 . 